\title{
Correction to: Phloretin reduces cell injury and inflammation mediated by Staphylococcus aureus via targeting sortase B and the molecular mechanism
}

\author{
Guizhen Wang ${ }^{1} \cdot$ Yawen Gao ${ }^{1} \cdot$ Hongsu Wang ${ }^{1} \cdot$ Jianfeng Wang ${ }^{2} \cdot$ Xiaodi Niu $^{1}$ \\ Published online: 31 May 2019 \\ (C) Springer-Verlag GmbH Germany, part of Springer Nature 2019
}

\section{Correction to: Applied Microbiology and Biotechnology} (2018) 102:10665-10674

https://doi.org/10.1007/s00253-018-9376-8

The images of cells under microscope in Figure 2 and Figure S2 were misused from Wang G et al. Front Cell Infect Microbiol. 2018 Nov 30;8:418. These images were generated in the same set of assays. We have repeated these experiments and got the consistent results, which have no influence on the results or conclusions in this study. The authors apologize for this error.

The online version of the original article can be found at https://oi.org/ 10.1007/s00253-018-9376-8

\footnotetext{
Jianfeng Wang wjf927@jlu.edu.cn

$\triangle$ Xiaodi Niu niuxd@jlu.edu.cn

1 College of Food Science and Engineering, Jilin University, Changchun, China

2 Key Laboratory of Zoonosis, Ministry of Education, College of Veterinary Medicine, Jilin University, Changchun, China
} 

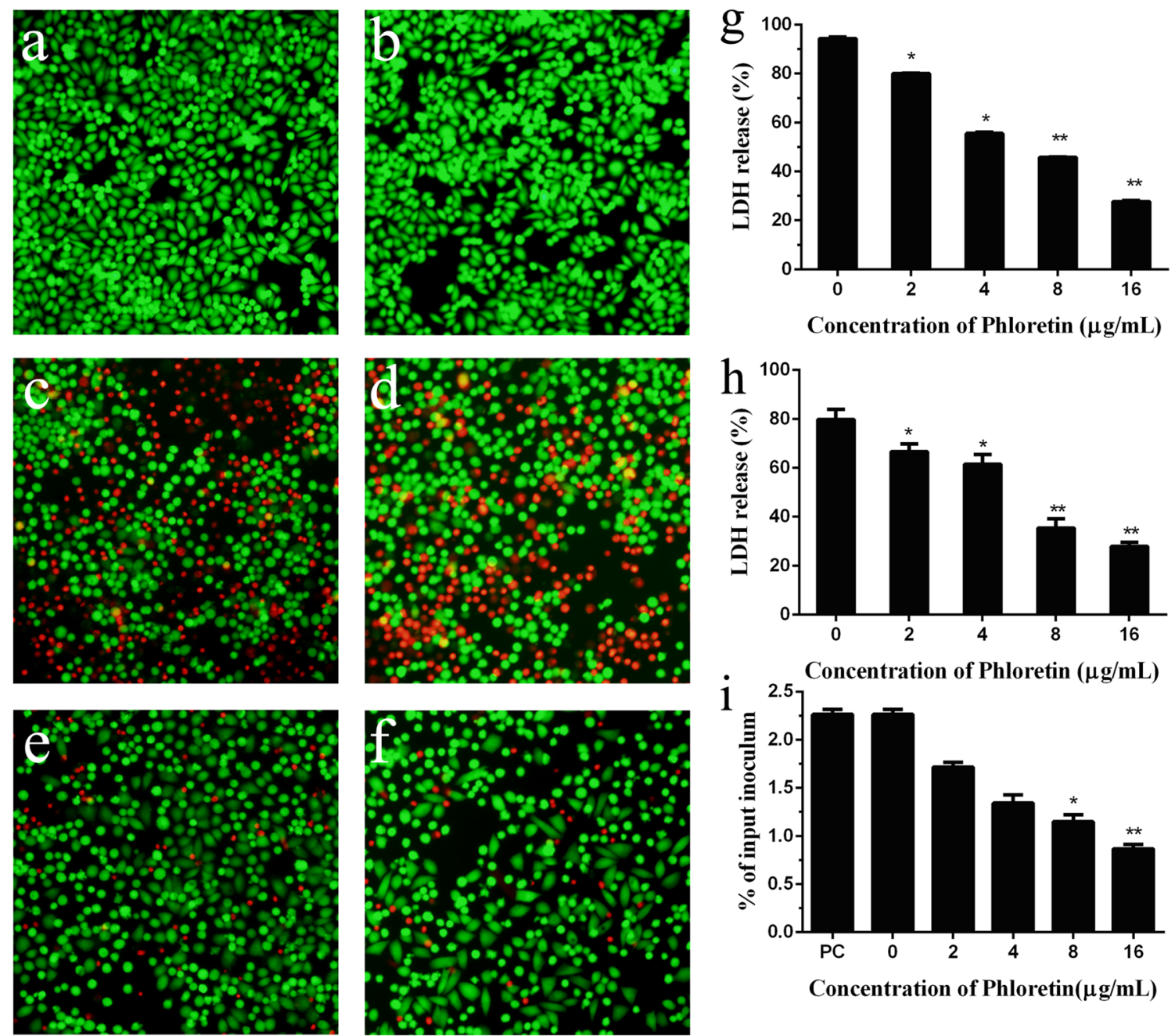

Fig. 2 Phloretin protects human alveolar epithelial cells from the injury caused by $S$. aureus and reduces the adhesion of $S$. aureus to human alveolar epithelial cells. The images of live (green)/dead (red) A549 cells were captured with a confocal laser scanning microscope. A549 cells uninfected with $S$. aureus (a and b). A549 cells infected with $S$. aureus 29213 (c) or Newman (d) but without phloretin. A549 cells treated with S. aureus 29213 (e) or Newman (f) in the presence of phloretin. LDH release from A549 cells treated with S. aureus 29213 (g) or Newman (h) and various concentrations of phloretin. i The adhesion levels of $S$. aureus 29213 to human alveolar epithelial cells, the results are presented as the percentage of colonies adhered to A549 cells and the initial inoculums size. The data were presented as means \pm $\mathrm{SD}(n=3) * *<0.05$ and $* * p<0.01$ 

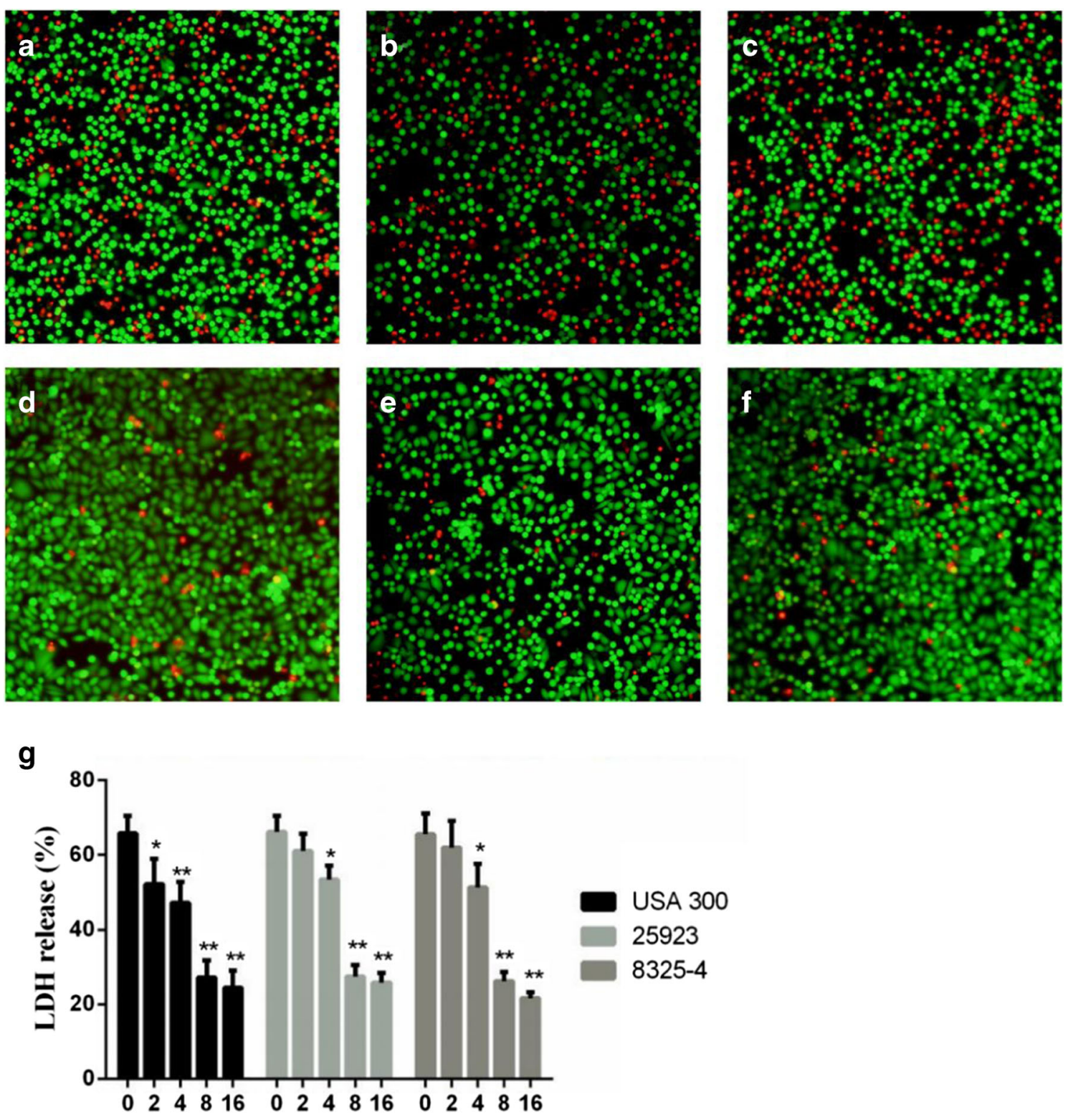

Concentration of Phloretin $(\mu \mathrm{g} / \mathrm{mL})$

Publisher's note Springer Nature remains neutral with regard to jurisdictional claims in published maps and institutional affiliations. 